\title{
Phase diagram of two-species Bose-Einstein condensates in an optical lattice
}

\author{
G. -P. Zheng ${ }^{1}$, J. -Q. Liang ${ }^{1}$, and W. M. Liu ${ }^{2}$ \\ 1. Institute of Theoretical Physics and Department of Physics, Shanxi \\ University, Taiyuan, Shanxi 030006, China \\ 2. Joint Laboratory of Advanced Technology in Measurements, Beijing National \\ Laboratory for Condensed Matter Physics, Institute of Physics, Chinese \\ Academy of Sciences, Beijing 100080, China
}

(November 5, 2018)

The exact macroscopic wave functions of two-species Bose-Einstein condensates in an optical lattice beyond the tight-binding approximation are studied by solving the coupled nonlinear Schrödinger equations. The phase diagram for superfluid and insulator phases of the condensates is determined analytically according to the macroscopic wave functions of the condensates, which are seen to be traveling matter waves.

PACS: 03.75.Mn, 03.75.Lm, 03.75.Kk

\section{INTRODUCTION}

Since the realization of Bose-Einstein condensates (BECs) in dilute atomic gases, a number of interesting experiments have been conducted to investigate multispecies Bose gases, in which two or more states of condensates exist together in a magnetic or optical trap $[1,2]$. Recently, vortex states have been obtained in a two-species Bose gas [3]. Progress in the experiments exploring dilute mixtures of quantum gases has stimulated intensive research on the properties of mixed Bose gases at zero temperature [4-6] and finite temperature as well [7].

The BEC trapped in an optical lattice exhibites a novel feature, namely the quantum phase transition between a Mott-insulator and a superfluid [8]. Such quantum phase tran- 
sition has attracted considerable attention in recent years. As a matter of fact, atomic gas of bosons in BEC subjected to a lattice potential which is turned on smoothly can be kept in the superfluid phase as long as the atom-atom interactions are small comparing with the tunnel coupling. In this regime, the kinetic energy is dominant in the total energy of the boson system. With an increase of the potential depth of the optical lattice, it is getting more and more difficult for bosons to tunnel from one site to the other, and finally the system attends an insulator phase above a critical value of the potential depth. In this case, the phase coherence is absent and the number of boson atoms in each lattice site becomes the same. The system possesses a Mott-insulator behavior. Various approaches have been proposed to understand theoretically the quantum phase transition and to determine the phase diagram as a function of BEC parameters [9-14].

Motivated by both the experimental and theoretical progress, we in the present paper study the phase diagram for superfluid and insulator phases of two-species BECs in a onedimensional (1D) optical lattice and the property of persistent current as well. The paper is organized as follows. In Sec. II, the exact macroscopic wave functions of the condensates which are not in the tight-binding regime are constructed by solving the coupled nonlinear Schrödinger equations. In Sec. III, the phase diagram is determined analytically according to the macroscopic wave functions of the condensates, i.e., the order parameters. Finally, we summarize our results in Sec. IV.

\section{THE EXACT MACROSCOPIC WAVE FUNCTIONS}

In this $1 \mathrm{D}$ geometry, the confinement along the radial direction is so tight that the trap frequency $\omega_{0}$ along the radial direction is much greater than the mean-field interaction energy. At low temperatures, the dynamics of the atoms in the radial direction is essentially "frozen," with all the atoms occupying the ground state of the harmonic trap with the wave function that

$$
\phi_{0}(y, z)=\sqrt{\frac{1}{\pi l_{0}^{2}}} \exp \left[-\left(y^{2}+z^{2}\right) / 2 l_{0}^{2}\right] .
$$


Here the extension of the wave function in the radial direction is given by the length scale $l_{0} \equiv \sqrt{\hbar / m \omega_{0}}$ of a harmonic oscillator, where $m$ is the mass of the atoms.

In the mean-field regime, $l_{0}$ is much greater than the radius of the interatomic potential $R_{e}$. The scattering of atoms in this effective 1D system is thus still a process of threedimension. According to ref. [15], the effective coupling constant in this 1D system is

$$
g_{1 D}=\frac{2 \hbar^{2}}{m} \frac{a}{l_{0}\left(l_{0}-C a\right)},
$$

where $a$ is the $s$-wave scattering length and $C$ is a numerical constant of the order unit. The term $C a$ in Eq. (2) is negligible for $l_{0}>>R_{e}$. In this limit, the expression for $g_{1 D}$ is the same as that obtained by averaging over the radial wave function (1),

$$
g_{1 D}=g_{3 D} \int_{-\infty}^{\infty} \int_{-\infty}^{\infty} d y d z \phi_{0}^{4}(y, z)=\frac{2 \hbar^{2} a}{m l_{0}^{2}} .
$$

We use this expression in the rest of this paper.

We consider the two-species BECs in a 1D periodic potential. The energy functional is seen to be

$$
\begin{aligned}
E\left[\psi_{1}, \psi_{2}\right]= & \int d x\left\{\sum _ { i = 1 , 2 } \left[\frac{\hbar^{2}}{2 m_{i}}\left|\frac{\partial \psi_{i}}{\partial x}\right|^{2}+V_{i}(x)\left|\psi_{i}\right|^{2}\right.\right. \\
& \left.\left.+\frac{\hbar^{2} a_{i}}{m_{i} l_{i}^{2}}\left|\psi_{i}\right|^{4}\right]+\frac{2 \hbar^{2} a_{12}}{\sqrt{m_{1} m_{2}} l_{1} l_{2}}\left|\psi_{1}\right|^{2}\left|\psi_{2}\right|^{2}\right\},
\end{aligned}
$$

where $\psi_{i}, m_{i}$, and $l_{i}=\sqrt{\hbar / m_{i} \omega_{0}}$ are the macroscopic wave functions of the condensates, the mass, and the harmonic-oscillator lengths in the radial direction of the $i$ th species $(i=1,2)$, respectively. $a_{1}, a_{2}$, and $a_{12}$ denote the $s$-wave scattering lengths between same-species and interspecies collisions. $V_{i}(x)$ are the periodic potentials,

$$
V_{i}(x)=V_{0, i} \operatorname{sn}^{2}\left(k_{L} x, k\right)
$$

with $V_{0, i}$ denoting the magnitude of potentials, where $k_{L}=2 \pi / \lambda$ is the wavevector of the laser light and $\lambda$ is the wavelength, corresponding to a lattice period $d=\lambda / 2 . \operatorname{sn}\left(k_{L} x, k\right)$ is the Jacobian elliptic sine function with modulus $k(0 \leq k \leq 1)$. In the limit $k=0$, the Jacobian elliptic sine reduces to the sinusoid function and thus $V(x)$ possesses a standard form 
of the standing light wave. For values of $k<0.9$, the potential is virtually indistinguishable from a standing light wave. Finally, for $k \rightarrow 1, V(x)$ becomes an array of well-separated hyperbolic secant potential barriers or wells.

The governing equations of the trapped BECs are obtained in terms of the variational procedure [16],

$$
i \hbar \frac{\partial \psi_{i}}{\partial t}=\frac{\delta E}{\delta \psi_{i}^{*}}
$$

which leads to the coupled nonlinear Schrödinger equations

$$
\begin{aligned}
i \hbar \frac{\partial \psi_{1}}{\partial t}= & -\frac{\hbar^{2}}{2 m_{1}} \frac{\partial^{2} \psi_{1}}{\partial x^{2}}+\frac{2 \hbar^{2} a_{1}}{m_{1} l_{1}^{2}}\left|\psi_{1}\right|^{2} \psi_{1} \\
& +\frac{2 \hbar^{2} a_{12}}{\sqrt{m_{1} m_{2}} l_{1} l_{2}}\left|\psi_{2}\right|^{2} \psi_{1}+V_{1}(x) \psi_{1}, \\
i \hbar \frac{\partial \psi_{2}}{\partial t}= & -\frac{\hbar^{2}}{2 m_{2}} \frac{\partial^{2} \psi_{2}}{\partial x^{2}}+\frac{2 \hbar^{2} a_{12}}{\sqrt{m_{1} m_{2}} l_{1} l_{2}}\left|\psi_{1}\right|^{2} \psi_{2} \\
& +\frac{2 \hbar^{2} a_{2}}{m_{2} l_{2}^{2}}\left|\psi_{2}\right|^{2} \psi_{2}+V_{2}(x) \psi_{2} .
\end{aligned}
$$

For the case of weakly coupled condensates in an optical lattice [17], the wave function $\psi$ can be decomposed as a sum of wave functions localized in each well of the periodic potential (tight-binding approximation) with the assumption relying on the fact that the height of the interwell barrier is much higher than the chemical potential. We, however, do not restrict ourselves to the low-energy case and look for the global condensate wave functions of excitations: $\psi_{i}(x, t)=\phi_{i}(x) \exp \left(-i \mu_{i} t / \hbar\right)$, where $\mu_{i}(i=1,2)$ are the chemical potentials. Thus the spatial wave functions satisfy the stationary coupled nonlinear Schrödinger equations that

$$
\begin{aligned}
\mu_{1} \phi_{1}= & -\frac{\hbar^{2}}{2 m_{1}} \frac{\partial^{2} \phi_{1}}{\partial x^{2}}+\frac{2 \hbar^{2} a_{1}}{m_{1} l_{1}^{2}}\left|\phi_{1}\right|^{2} \phi_{1} \\
& +\frac{2 \hbar^{2} a_{12}}{\sqrt{m_{1} m_{2}} l_{1} l_{2}}\left|\phi_{2}\right|^{2} \phi_{1}+V_{1}(x) \phi_{1}, \\
\mu_{2} \phi_{2}= & -\frac{\hbar^{2}}{2 m_{2}} \frac{\partial^{2} \phi_{2}}{\partial x^{2}}+\frac{2 \hbar^{2} a_{12}}{\sqrt{m_{1} m_{2}} l_{1} l_{2}}\left|\phi_{1}\right|^{2} \phi_{2} \\
& +\frac{2 \hbar^{2} a_{2}}{m_{2} l_{2}^{2}}\left|\phi_{2}\right|^{2} \phi_{2}+V_{2}(x) \phi_{2} .
\end{aligned}
$$


With the general form of spatial wave functions $\phi_{i}(x)$ written as [18] $\phi_{i}(x)=$ $r_{i}(x) \exp \left[i \varphi_{i}(x)\right]$, Eq. (8) can be separated as real and imaginary parts. We then integrate once for the imaginary part and obtain the first-order differential equations for the phases $\varphi_{i}(x)$,

$$
\varphi_{i}^{\prime}(x)=\frac{\alpha_{i}}{r_{i}^{2}(x)},
$$

where parameters $\alpha_{i}(i=1,2)$ are constants of integration to be determined. Substituting Eq. (9) into the real part obtained from Eq. (8) and integrating again, we find

$$
\begin{aligned}
\left(r_{1} r_{1}^{\prime}\right)^{2}= & \frac{2 a_{1}}{l_{1}^{2}} r_{1}^{6}-\frac{2 m_{1} \mu_{1}}{\hbar^{2}} r_{1}^{4}+\beta_{1} r_{1}^{2}-\alpha_{1}^{2} \\
& +\frac{4 a_{12} \sqrt{m_{1}}}{\sqrt{m_{2}} l_{1} l_{2}} r_{1}^{2} \int r_{2}^{2} d\left(r_{1}^{2}\right) \\
& +\frac{2 m_{1}}{\hbar^{2}} r_{1}^{2} \int V_{1}(x) d\left(r_{1}^{2}\right), \\
\left(r_{2} r_{2}^{\prime}\right)^{2}= & \frac{2 a_{2}}{l_{2}^{2}} r_{2}^{6}-\frac{2 m_{2} \mu_{2}}{\hbar^{2}} r_{2}^{4}+\beta_{2} r_{2}^{2}-\alpha_{2}^{2} \\
& +\frac{4 a_{12} \sqrt{m_{2}}}{\sqrt{m_{1}} l_{1} l_{2}} r_{2}^{2} \int r_{1}^{2} d\left(r_{2}^{2}\right) \\
& +\frac{2 m_{2}}{\hbar^{2}} r_{2}^{2} \int V_{2}(x) d\left(r_{2}^{2}\right),
\end{aligned}
$$

where $\beta_{i}(i=1,2)$ denote additional constants of integration.

We then construct the solutions as

$$
r_{i}^{2}(x)=A_{i} \operatorname{sn}^{2}\left(k_{L} x, k\right)+B_{i},
$$

where the constants $B_{i}(i=1,2)$ determine the mean amplitudes and act as the dc offsets for the numbers of the condensed atoms [19], and the parameters $A_{i}(i=1,2)$ are to be determined.

Substituting Eq. (11) into Eq. (10) and using identities of Jacobian elliptic functions, we obtain eight equations for the parameters $\alpha_{i}, \beta_{i}, \mu_{i}$, and $A_{i}$. Eliminating $\beta_{i}$, we find

$$
\begin{aligned}
& A_{1}=\frac{\frac{\sqrt{m_{1}} l_{1} l_{2} a_{12}}{\sqrt{m_{2}}}\left(m_{2} V_{0,2}-\hbar^{2} k_{L}^{2} k^{2}\right)-a_{2} l_{1}^{2}\left(m_{1} V_{0,1}-\hbar^{2} k_{L}^{2} k^{2}\right)}{2 \hbar^{2}\left(a_{1} a_{2}-a_{12}^{2}\right)}, \\
& A_{2}=\frac{\frac{\sqrt{m_{2}} l_{1} l_{2} a_{12}}{\sqrt{m_{1}}}\left(m_{1} V_{0,1}-\hbar^{2} k_{L}^{2} k^{2}\right)-a_{1} l_{2}^{2}\left(m_{2} V_{0,2}-\hbar^{2} k_{L}^{2} k^{2}\right)}{2 \hbar^{2}\left(a_{1} a_{2}-a_{12}^{2}\right)},
\end{aligned}
$$


and

$$
\begin{aligned}
& \alpha_{1}^{2}=B_{1} k_{L}^{2}\left[\frac{k^{2}}{A_{1}} B_{1}^{2}+\left(1+k^{2}\right) B_{1}+A_{1}\right], \\
& \alpha_{2}^{2}=B_{2} k_{L}^{2}\left[\frac{k^{2}}{A_{2}} B_{2}^{2}+\left(1+k^{2}\right) B_{2}+A_{2}\right],
\end{aligned}
$$

and

$$
\begin{aligned}
\mu_{1}= & \frac{\hbar^{2} k_{L}^{2}}{2 m_{1}}\left(1+k^{2}+\frac{6 a_{1}}{l_{1}^{2} k_{L}^{2}} B_{1}+\frac{4 a_{12} \sqrt{m_{1}}}{l_{1} l_{2} k_{L}^{2} \sqrt{m_{2}}} B_{2}\right. \\
& \left.+\frac{2 a_{12} \sqrt{m_{1}}}{l_{1} l_{2} k_{L}^{2} \sqrt{m_{2}}} \frac{A_{2}}{A_{1}} B_{1}+\frac{m_{1} V_{0,1}}{\hbar^{2} k_{L}^{2}} \frac{B_{1}}{A_{1}}\right), \\
\mu_{2}= & \frac{\hbar^{2} k_{L}^{2}}{2 m_{2}}\left(1+k^{2}+\frac{6 a_{2}}{l_{2}^{2} k_{L}^{2}} B_{2}+\frac{4 a_{12} \sqrt{m_{2}}}{l_{1} l_{2} k_{L}^{2} \sqrt{m_{1}}} B_{1}\right. \\
& \left.+\frac{2 a_{12} \sqrt{m_{2}}}{l_{1} l_{2} k_{L}^{2} \sqrt{m_{1}}} \frac{A_{1}}{A_{2}} B_{2}+\frac{m_{2} V_{0,2}}{\hbar^{2} k_{L}^{2}} \frac{B_{2}}{A_{2}}\right) .
\end{aligned}
$$

For $k=0, \operatorname{sn}\left(k_{L} x, 0\right)=\sin \left(k_{L} x\right)$, the solutions reduce to

$$
\psi_{i}(x, t)=\sqrt{A_{i}^{0} \sin ^{2}\left(k_{L} x\right)+B_{i}} \exp \left\{i\left[\varphi_{i}^{0}(x)-\mu_{i}^{0} t / \hbar\right]\right\}
$$

where

$$
\begin{aligned}
& A_{1}^{0}=\frac{\sqrt{m_{1} m_{2}} a_{12} l_{1} l_{2} V_{0,2}-m_{1} a_{2} V_{0,1} l_{1}^{2}}{2 \hbar^{2}\left(a_{1} a_{2}-a_{12}^{2}\right)}, \\
& A_{2}^{0}=\frac{\sqrt{m_{2} m_{1}} a_{12} l_{1} l_{2} V_{0,1}-m_{2} a_{1} V_{0,2} l_{2}^{2}}{2 \hbar^{2}\left(a_{1} a_{2}-a_{12}^{2}\right)} .
\end{aligned}
$$

The phases $\varphi_{i}^{0}(x)(i=1,2)$ are determined by nonlinear equations

$$
\tan \left[\varphi_{i}^{0}(x)\right]= \pm \sqrt{1+\frac{A_{i}^{0}}{B_{i}}} \tan \left(k_{L} x\right)
$$

and

$$
\begin{aligned}
\mu_{1}^{0}= & \frac{\hbar^{2} k_{L}^{2}}{2 m_{1}}\left(1+\frac{6 a_{1}}{l_{1}^{2} k_{L}^{2}} B_{1}+\frac{4 a_{12} \sqrt{m_{1}}}{l_{1} l_{2} k_{L}^{2} \sqrt{m_{2}}} B_{2}\right. \\
& \left.+\frac{2 a_{12} \sqrt{m_{1}}}{l_{1} l_{2} k_{L}^{2} \sqrt{m_{2}}} \frac{A_{2}^{0}}{A_{1}^{0}} B_{1}+\frac{m_{1} V_{0,1}}{\hbar^{2} k_{L}^{2}} \frac{B_{1}}{A_{1}^{0}}\right), \\
\mu_{2}^{0}= & \frac{\hbar^{2} k_{L}^{2}}{2 m_{2}}\left(1+\frac{6 a_{2}}{l_{2}^{2} k_{L}^{2}} B_{2}+\frac{4 a_{12} \sqrt{m_{2}}}{l_{1} l_{2} k_{L}^{2} \sqrt{m_{1}}} B_{1}\right. \\
& \left.+\frac{2 a_{12} \sqrt{m_{2}}}{l_{1} l_{2} k_{L}^{2} \sqrt{m_{1}}} \frac{A_{1}^{0}}{A_{2}^{0}} B_{2}+\frac{m_{2} V_{0,2}}{\hbar^{2} k_{L}^{2}} \frac{B_{2}}{A_{2}^{0}}\right) .
\end{aligned}
$$

The constants $A$ and $B$ are related by restrictions such that $B_{1} \geq-A_{1}^{0}$ for $A_{1}^{0}<0$ and $B_{1} \geq 0$ for $A_{1}^{0}>0 ; B_{2} \geq-A_{2}^{0}$ for $A_{2}^{0}<0$ and $B_{2} \geq 0$ for $A_{2}^{0}>0$. 


\section{THE PHASE DIAGRAM}

The average particle number densities $n_{i}$ for the two species are obtained as

$$
\begin{aligned}
n_{i} & =\frac{1}{L} \int_{0}^{L}\left|\psi_{i}(x, t)\right|^{2} d x \\
& =\frac{1}{h \pi} \int_{0}^{h \pi}\left[A_{i}^{0} \sin ^{2}\left(x^{\prime}\right)+B_{i}\right] d x^{\prime}
\end{aligned}
$$

where $x^{\prime}=k_{L} x$ and $L=h d$ denotes the length of the optical lattice with $h=1,2,3, \ldots$ This leads to

$$
B_{i}=n_{i}-\frac{A_{i}^{0}}{2}
$$

Then the macroscopic wave functions of the condensates Eq. (15) can exist only when

$$
\begin{aligned}
& n_{1} \geq \frac{\left|A_{1}^{0}\right|}{2}=\frac{\left|a_{12} V_{0,2}-a_{2} V_{0,1}\right|}{4 \hbar \omega_{0}\left|a_{1} a_{2}-a_{12}^{2}\right|}, \\
& n_{2} \geq \frac{\left|A_{2}^{0}\right|}{2}=\frac{\left|a_{12} V_{0,1}-a_{1} V_{0,2}\right|}{4 \hbar \omega_{0}\left|a_{1} a_{2}-a_{12}^{2}\right|} .
\end{aligned}
$$

The condensate atom currents can be evaluated from the usual definition, $j=$ $(\hbar / m) \operatorname{Im}\left[\psi^{*}(\partial \psi / \partial x)\right][20]$, with the exact wave functions Eq. (15) which are seen to be travelling matter waves. The result is

$$
\begin{aligned}
j_{i} & = \pm \frac{\hbar k_{L}}{m_{i}} \sqrt{B_{i}\left(B_{i}+A_{i}^{0}\right)} \\
& = \pm \frac{\hbar k_{L}}{m_{i}} \sqrt{n_{i}^{2}-\frac{\left(A_{i}^{0}\right)^{2}}{4}}
\end{aligned}
$$

which are independent of space-time variables and therefore persistent currents. We may demand that the wave functions Eq. (15) satisfy the periodic boundary condition $\psi_{i}(x, t)=$ $\psi_{i}(x+L, t)$ which is naturally fulfilled as the total length $L$ is an integer times the lattice constant $d(L=h d, h=1,2,3, \ldots)$. These periodic solutions in $1 \mathrm{D}$ space with spatial period $L$ are equivalent to the solutions in a ring of circumstance $L$. The persistent currents then can be viewed as in the optical lattice ring.

It is found from Eq. (22) that the persistent currents are valid only for conditions in which the number density of atoms is greater than critical values, $n_{i} \geq\left|A_{i}^{0}\right| / 2$, i.e., when the 
macroscopic wave functions of the condensates exist. These persistent currents are similar to the 1D Fröhlich superconductivity induced by the traveling lattice wave [21] and can be controlled by adjusting the barriers height of the periodic potentials and parameters of the bosonic atoms. The currents increase with the decrease of $\left|A_{i}^{0}\right|$ and approach the asymptotic maximum values $j_{i, \max }= \pm \hbar n_{i} k_{L} / m_{i}$ when $\left|A_{i}^{0}\right|$ become vanishingly small. With the recent progress made on confinement of atoms in the light-induced $[22,23]$ as well as the magnetic-field-induced [24,25] atom waveguides [26], the persistent currents may be observed experimentally in the future.

The energy spectrum for the two species is obtained as

$$
\begin{aligned}
& \mu_{1}^{0}=E_{R, 1}+\frac{V_{0,1}}{2}+2 \hbar \omega_{0}\left(a_{1} n_{1}+a_{12} n_{2}\right), \\
& \mu_{2}^{0}=E_{R, 2}+\frac{V_{0,2}}{2}+2 \hbar \omega_{0}\left(a_{2} n_{2}+a_{12} n_{1}\right),
\end{aligned}
$$

where $E_{R, i}=\hbar^{2} k_{L}^{2} / 2 m_{i}$ are the recoil energy of an atom absorbing one of the lattice phonons $[17]$.

In this paper, we consider the two species both with repulsive interactions, namely, $a_{1}>0, a_{2}>0, a_{12}>0$. Then the macroscopic wave functions of the condensates can exist only when

$$
\begin{aligned}
\mu_{1}^{0} \geq & E_{R, 1}+\frac{V_{0,1}}{2}+\frac{a_{1}\left|a_{12} V_{0,2}-a_{2} V_{0,1}\right|}{2\left|a_{1} a_{2}-a_{12}^{2}\right|} \\
& +\frac{a_{12}\left|a_{12} V_{0,1}-a_{1} V_{0,2}\right|}{2\left|a_{1} a_{2}-a_{12}^{2}\right|}, \\
\mu_{2}^{0} \geq & E_{R, 2}+\frac{V_{0,2}}{2}+\frac{a_{2}\left|a_{12} V_{0,1}-a_{1} V_{0,2}\right|}{2\left|a_{1} a_{2}-a_{12}^{2}\right|} \\
& +\frac{a_{12}\left|a_{12} V_{0,2}-a_{2} V_{0,1}\right|}{2\left|a_{1} a_{2}-a_{12}^{2}\right|} .
\end{aligned}
$$

The macroscopic wave functions of the condensates $\psi_{i}(x, t)$ are complex functions defined as the expectation value of the boson field operators: $\psi_{i}(x, t) \equiv\left\langle\hat{\Psi}_{i}(x, t)\right\rangle$, which have the meaning of order parameters and characterize the off-diagonal long-range behavior of the one-particle density matrix $\rho_{i}\left(x^{\prime}, x, t\right)=\left\langle\hat{\Psi}_{i}^{+}\left(x^{\prime}, t\right) \hat{\Psi}_{i}(x, t)\right\rangle[16]$. So the condensates can be kept in the superflud phase only when the macroscopic wave functions exist. Otherwise, the 
phase coherence and the currents vanish and therefore the condensates are in the insulator phase. Then we obtain four cases of phases for two-species BECs in a 1D optical lattice as follows.

Case 1. The two species are both in the superfluid phase, namely

$$
\begin{aligned}
& n_{1} \geq \frac{\left|a_{12} V_{0,2}-a_{2} V_{0,1}\right|}{4 \hbar \omega_{0}\left|a_{1} a_{2}-a_{12}^{2}\right|}, \\
& n_{2} \geq \frac{\left|a_{12} V_{0,1}-a_{1} V_{0,2}\right|}{4 \hbar \omega_{0}\left|a_{1} a_{2}-a_{12}^{2}\right|},
\end{aligned}
$$

which is labeled as SS in the phase diagram, Fig. 1.

Case 2. Species 1 is in the superfluid phase while species 2 is in the insulator phase,

$$
\begin{aligned}
& n_{1} \geq \frac{\left|a_{12} V_{0,2}-a_{2} V_{0,1}\right|}{4 \hbar \omega_{0}\left|a_{1} a_{2}-a_{12}^{2}\right|}, \\
& n_{2} \leq \frac{\left|a_{12} V_{0,1}-a_{1} V_{0,2}\right|}{4 \hbar \omega_{0}\left|a_{1} a_{2}-a_{12}^{2}\right|},
\end{aligned}
$$

labeled as SI.

Case 3. Species 1 is in the insulator phase and species 2 is in the superfluid phase,

$$
\begin{aligned}
& n_{1} \leq \frac{\left|a_{12} V_{0,2}-a_{2} V_{0,1}\right|}{4 \hbar \omega_{0}\left|a_{1} a_{2}-a_{12}^{2}\right|}, \\
& n_{2} \geq \frac{\left|a_{12} V_{0,1}-a_{1} V_{0,2}\right|}{4 \hbar \omega_{0}\left|a_{1} a_{2}-a_{12}^{2}\right|},
\end{aligned}
$$

labeled as IS.

Case 4. The two species are both in the insulator phase,

$$
\begin{aligned}
& n_{1} \leq \frac{\left|a_{12} V_{0,2}-a_{2} V_{0,1}\right|}{4 \hbar \omega_{0}\left|a_{1} a_{2}-a_{12}^{2}\right|}, \\
& n_{2} \leq \frac{\left|a_{12} V_{0,1}-a_{1} V_{0,2}\right|}{4 \hbar \omega_{0}\left|a_{1} a_{2}-a_{12}^{2}\right|},
\end{aligned}
$$

labeled as II.

The quantum phases of the condensates can be determined by all parameters of BECs and optical lattice as shown above. In Fig. 1, we show the phase diagram with the various same-species $s$-wave scattering lengths and equal particle number density $n_{1}=n_{2}=n$ and 
the magnitudes of potentials $\left(V_{0,1}=V_{0,2}=V_{0}\right)$ for simplicity. Thus the conditions for the two species in the superfluid phase are given by

$$
\frac{V_{0}}{4 n \hbar \omega_{0}} \leq \frac{\left|a_{1} a_{2}-a_{12}^{2}\right|}{\left|a_{12}-a_{2}\right|}
$$

and

$$
\frac{V_{0}}{4 n \hbar \omega_{0}} \leq \frac{\left|a_{1} a_{2}-a_{12}^{2}\right|}{\left|a_{12}-a_{1}\right|}
$$

respectively.

Component separation in two-species BECs has been predicted by means of mean-field theory [4-7] and observed in experiments $[1,2]$ when the relation of the scattering lengths that $a_{12}>\sqrt{a_{1} a_{2}}$ is fulfilled. From the above conditions Eqs. (29), (30) and the phase diagram Fig. 1, we find that the larger values of $a_{12}$ favor the superfluid phase in the twospecies mixture and the component separation according to the experimental observation $[1,2]$. Particularly when the interspecies scattering length approaches the value of the samespecies such that $a_{12}=a_{2}$ or $a_{1}$, the conditions Eqs. $\left.(29), 30\right)$ result in the superfluid phase independent of the potential magnitude $V_{0}$. One should not be surprised by this result since we consider the case in which the chemical potential is always higher than the potential magnitude $V_{0}$ seen from Eq. (24).

\section{CONCLUSION}

In conclusion, the exact macroscopic wave functions of two-species BECs in an optical lattice beyond the tight-binding approximation are studied. The phase diagram is determined analytically according to the order parameters, and persistent currents in an optical lattice ring are obtained explicitly in terms of the exact wave functions, which are seen to be traveling matter waves. 


\section{ACKNOWLEDGMENTS}

This work was supported by the NSF of China under Grants No. 10475053, No. 60490280, No. 90406017, and No. 90403034.

[1] C.J. Myatt et al., Phys. Rev. Lett. 78, 586 (1997); M.R. Matthews et al., ibid. 81, 243 (1998); D.S. Hall et al., ibid. 81, 1539 (1998); ibid. 81, 1543 (1998); G. Modugno et al., Science 294, 1320 (2001); Phys. Rev. Lett. 89, 190404 (2002).

[2] D.M. Stamper-Kurn et al., Phys. Rev. Lett. 80, 2027 (1998); H.J. Miesner et al., ibid. 82, 2228 (1999); D.M. Stamper-Kurn et al., ibid. 83, 661 (1999); W.M. Liu et al., ibid. 84, 2294 (2000); 88, 170408 (2002).

[3] M.R. Matthews, B.P. Anderson, P.C. Haljan, D.S. Hall, C.E. Wieman, and E.A. Cornell, Phys. Rev. Lett. 83, 2498 (1999).

[4] T.L. Ho and V.B. Shenoy, Phys. Rev. Lett. 77, 3276 (1996).

[5] B.D. Esry, C.H. Greene, J.P. Burke, and J.L. Bohn, Phys. Rev. Lett. 78, 3594 (1997).

[6] H. Pu and N.P. Bigelow, Phys. Rev. Lett. 80, 1130 (1998).

[7] H.L. Shi, W.M. Zheng, and S.T. Chui, Phys. Rev. A 61, 063613 (2000).

[8] M. Greiner, O. Mandel, T. Esslinger, T.W. Hänsch, and I. Bloch, Nature (London) 415, 39 (2002).

[9] M.P.A. Fisher, P.B. Weichman, G. Grinstein, and D.S. Fisher, Phys. Rev. B 40, 546 (1989).

[10] D. Jaksch, C. Bruder, J. I. Cirac, C. W. Gardiner, and P. Zoller, Phys. Rev. Lett. 81, 3108 (1998).

[11] D. van Oosten, P. van der Straten, and H.T.C. Stoof, Phys. Rev. A 63, 053601 (2001). 
[12] G.-H. Chen and Y.-S. Wu, Phys. Rev. A 67, 013606 (2003).

[13] J.-J. Liang, J.-Q. Liang, and W.-M. Liu, Phys. Rev. A 68, 043605 (2003).

[14] Z.-W. Xie and W.M. Liu, Phys. Rev. A 70, 045602 (2004).

[15] M. Olshanii, Phys. Rev. Lett. 81, 938 (1998).

[16] F. Dalfovo, S. Giorgini, L.P. Pitaevskii, and S. Stringari, Rev. Mod. Phys. 71, 463 (1999).

[17] F.S. Cataliotti, S. Berger, C. Fort, P. Maddaloni, F. Minardi, A. Trombrttoni, A. Smerzi, and M. Inguscio, Science 293, 843 (2001).

[18] L.D. Carr, C.W. Clark, and W.P. Reinhardt, Phys. Rev. A 62, 063610 (2000).

[19] J.C. Bronski, L.D. Carr, B. Deconinck, and J.N. Kutz, Phys. Rev. Lett. 86, 1402 (2001).

[20] D.I. Choi and Q. Niu, Phys. Rev. Lett. 82, 2022 (1999); G.-P. Zheng and J.-Q. Liang, Commun. Theor. Phys. 43 (5), 819 (2005).

[21] H. Fröhlich, Proc. R. Soc. London, Ser. A 223, 296 (1954).

[22] M.A. Ol'shanii, Y.B. Ovchinnikov, and V.S. Letokhov, Opt. Commun. 98, 77 (1993).

[23] M.J. Renn, D. Montgomery, O. Vdovin, D.Z. Anderson, C.E. Wieman, and E.A. Cornell, Phys. Rev. Lett. 75, 3253 (1995).

[24] J. Denschlag, D. Cassettari, and J. Schmiedmayer, Phys. Rev. Lett. 82, 2014 (1999).

[25] A.E. Leanhardt, A.P. Chikkatur, D. Kielpinski, Y. Shin, T.L. Gustavson, W. Ketterle, and D.E. Pritchard, Phys. Rev. Lett. 89, 040401 (2002).

[26] M.A. Kasevich, Science 298, 1363 (2002). Figure Caption:

FIG. 1. Phase diagrams of two-species BECs in a 1D optical lattice. The magnitude of potentials $V_{0}$ is in units of $4 n \hbar \omega_{0}$. The interspecies scattering length is in units of $n m$. Dashed 
curves: phase boundary of species 1 ; solid curves: phase boundary of species 2 . The $s$-wave scattering lengths between the same-species are as follows: (a) $a_{1}=6 \mathrm{~nm}\left({ }^{87} \mathrm{Rb}\right), a_{2}=3 \mathrm{~nm}$ $\left({ }^{23} \mathrm{Na}\right)$. (b) $a_{1}=3 \mathrm{~nm}$ (hyperfine state $\left|f=1, m_{f}=1\right\rangle$ of $\left.{ }^{23} \mathrm{Na}\right), a_{2}=2.5 \mathrm{~nm}$ (hyperfine state $\left|f=1, m_{f}=0\right\rangle$ of $\left.{ }^{23} \mathrm{Na}\right)$. 
Fig. 1a

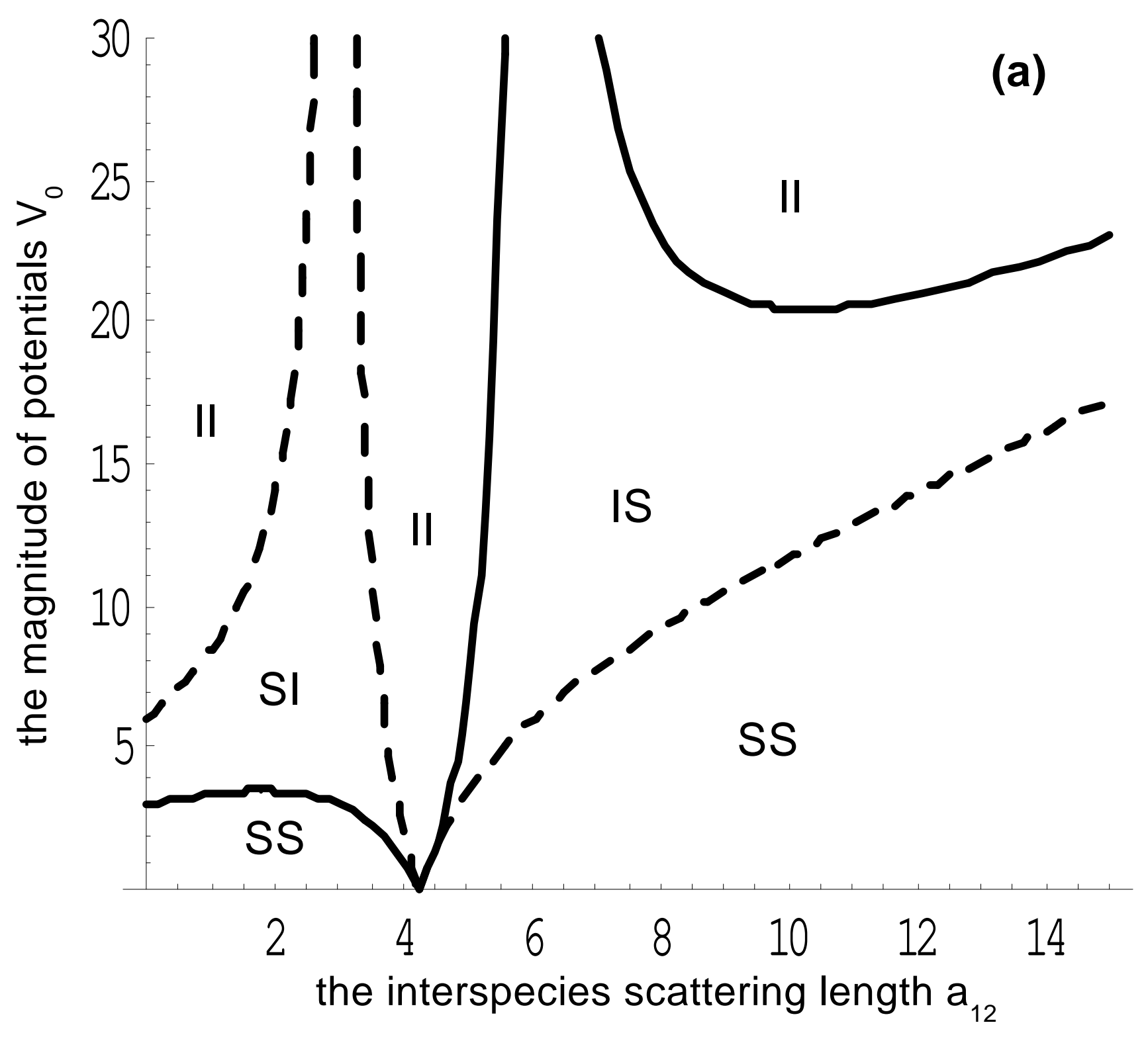


Fig. 1b

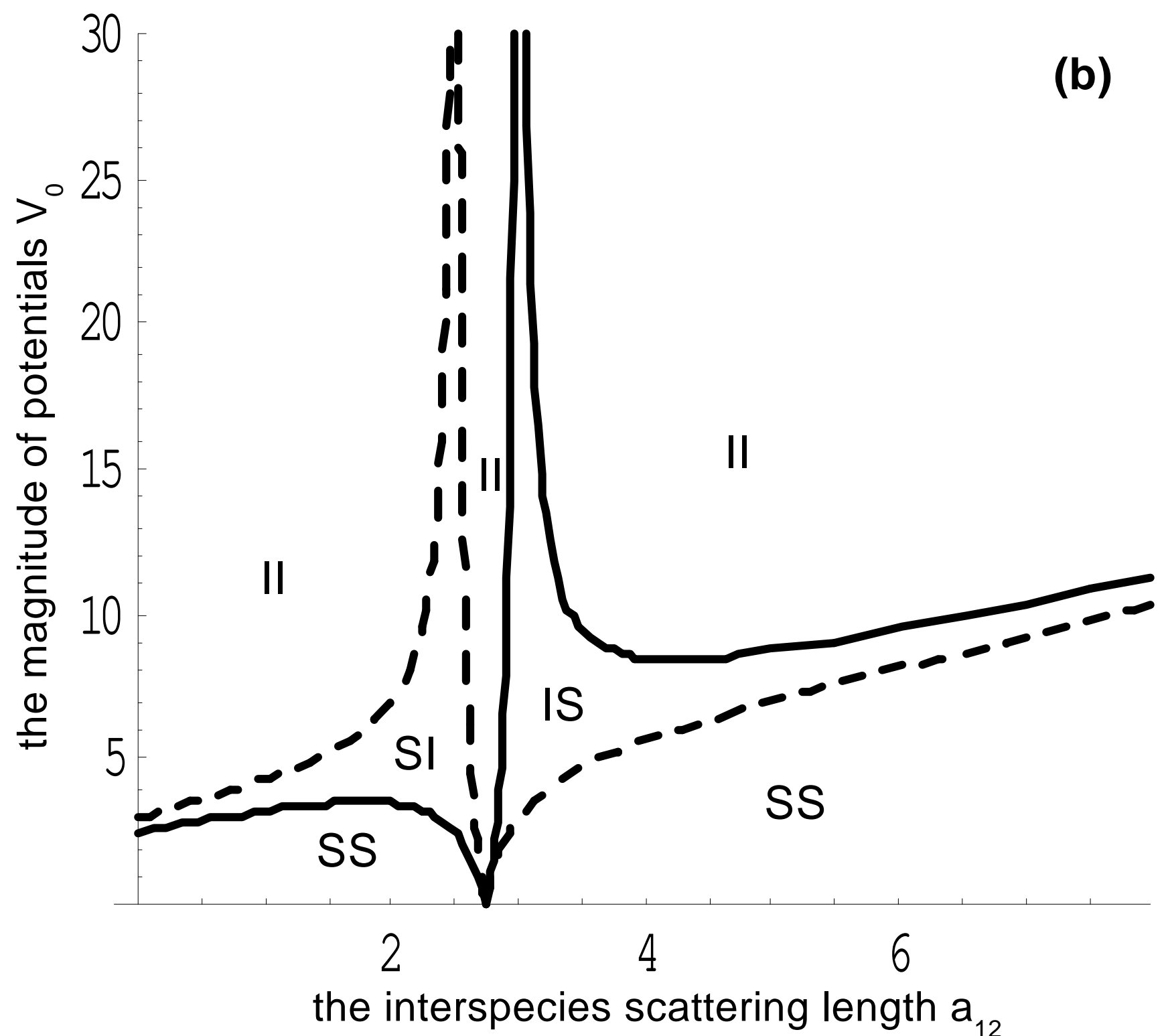

